\title{
Philosophiques
}

\section{La parole risquée}

\section{Patrick Drevet}

Volume 22, numéro 1, printemps 1995

URI : https://id.erudit.org/iderudit/027312ar

DOI : https://doi.org/10.7202/027312ar

Aller au sommaire du numéro

Éditeur(s)

Société de philosophie du Québec

ISSN

0316-2923 (imprimé)

1492-1391 (numérique)

Découvrir la revue

Citer cet article

Drevet, P. (1995). La parole risquée. Philosophiques, 22(1), 103-115.

https://doi.org/10.7202/027312ar

Ce document est protégé par la loi sur le droit d'auteur. L'utilisation des services d'Érudit (y compris la reproduction) est assujettie à sa politique d'utilisation que vous pouvez consulter en ligne.

https://apropos.erudit.org/fr/usagers/politique-dutilisation/
Cet article est diffusé et préservé par Érudit.

Érudit est un consortium interuniversitaire sans but lucratif composé de l'Université de Montréal, l'Université Laval et l'Université du Québec à Montréal. Il a pour mission la promotion et la valorisation de la recherche. https://www.erudit.org/fr/ 


\title{
LA PAROLE RISQUUÉ
}

\author{
par \\ Patrick Drevet
}

Qui n'a pas au moins une fois reçu une sorte d'illumination en prononçant ou en entendant un mot qui, pour ne lui être pas inconnu et pouvant passer même pour ordinaire, lui a paru donner soudain, avec une acuité et une profondeur renversantes, toute sa dimension à son sens? Mot enchâssé dans une expression qu'il avait repétée jusque là sans y penser; mot dont il n'avait jamais pesé le poids dans le vers d'un poème reste en sa mémoire depuis l'enfance; mot dans un titre qu'une fois lu le contenu de l'ouvrage qui le porte dote d'une force d'évocation immense; mot dont la place que l'écrivain lui a choisie exalte la signification jusqu'à lui donner le pouvoir d'un césame, le révèle clef des songes...

Il n'est pas besoin que ce mot soit rare. Je le connaissais et l'employais communément, je ne l'avais jamais êprouvé comme cela. Voici qu'il irradie, isolè, porteur à lui seul de l'univers, éclatant de signification et en même temps fascinant comme une énigme. Ce mot commun prend la résonance alarmante d'un nom propre. Je suis surpris par lui : il n'a pas seulement le sens que je pourrais trouver explicite dans le dictionnaire, il est épais, vivant, vibrant d'expérience, il plonge au cour de son origine que je ne connais pas et il m'arrive avec tout le fleuve de significations qu'ont grossi un a un ceux qui l' ont employé avant moi, tel qu'en lui-même, avec l'éclat de l'évidence. Il n'était qu'un mot flou dans la pâte du langage : il s'est avancé jusqu'à moi et je me suis trouvé moi-même, à ce moment là, dans les dispositions requises pour le découvirir, détaché de la masse informe du vocabulaire, rayonnant de sa personnalité propre, comme s'il s'était fait chair. Il me place dans un rapport nouveau avec le monde encore qu'il indique que ce rapport qui me semble nouveau est précisément ce qui avait toujours été. Ce n'est pas au lexique qu'il me donne accès mais à l'humanité. Ce mot enfin « compris » m'implique dans ma sensibilité et dans mon histoire : il m'exprime d'une façon si violente que pour un peuj'en rougis. 
Il semble que les auteurs anciens avaient beaucoup plus ordinairement que nous cette attention au pouvoir illuminant des mots, et un sens plus aigu des implications que leur profération est susceptible d'engendrer. Les lire plonge dans un âge ou l'homme plus silencieux n'avançait dans ses phrases qu'à pas comptés, prenant le temps de choisir ses mots, de les soupeser, de les lâcher avec la réticence qu'inspire la conscience de la valeur de ce que l'on donne. Cela frappe encore chez les auteurs classiques : les discours des héros de La Princesse de Clèves, les périphrases de Chimène, les échappatoires de Célimène, les litotes de Bérénice ou de Phèdre alors qu'elles sont toujours mises en demeure d'avouer témoignent d'une terreur sacrée à l'endroit du langage. Le héros classique le sait : dès qu'il a prononcé le mot qui définit ce qu'il n'est pas encore tout en l'étant déjà, il ne peut plus s'en defaire. Créature de langage, il n'a pas les ressources du repentir ou de la rature, il ne lui est pas permis de gommer les mots qui le constituent à mesure qu'il les prononce, ils sont sa fatalité.

Il nous en reste quelque chose au croisement des mots et de la mort, à celui des mots et de l'amour. Nous évitons encore, comme les Grecs et les Latins, d'employer le mot « mort » pour évoquer la réalité qu'il désigne : nous disons décéder (decedere e vita, descendre de la vie); nous disons passer, trépasser (interire, passer à travers); nous disons «n'être plus ». Mais d'où nous vient la crainte souvent insurmontable qui nous rend si difficile aussi la prononciation d'un «je t'aime» sinon de la conscience aiguë que nous avons alors du sens et de l'engagement que ce mot soudain représente? Par lui nous ne traduisons pas ce qui est, nous ne représentons pas un état d'âme, nous ne certifions pas davantage : nous créons. Le mot nous verse dans un réel auquel, tout en l'imaginant, nous n'avions pas songé avant d'être sur le point de le prononcer. Nous nous rendons compte seulement de la situation, presque oppressante à force d'épaisseur et de réalité, dans laquelle il suffit que nous le proférions pour que nous soyons mis. Par ce mot qui, jusqu'alors, ne traduisait qu'une impression, une idée, le désir, nous ne sommes plus isolés sans doute mais nous touchons à une solitude et à une nudité qui nous rendent au plus haut point vulnérables. Nous ne dépendons plus uniquement de mots, d'images, de fantasmes, c'est-à-dire de nous-mêmes : nous nous exposons à la sanction du monde et à la réponse de l'autre auxquels échoit de réaliser ou non notre possible.

\section{II}

A chaque fois que, par une conjonction plus ou moins hasardeuse de conditions favorables, mesurant l'importance cruciale de la profération des mots sur le cours de notre existence, découvrant qu'ils détiennent avant nous le mystère de notre inscription dans le monde, nous prenons conscience non seulement de la valeur d'un mot mais encore de la profondeur et de l'étendue 
du réel qu'il engage, nous quittons le domaine du langage et de la communication pour entrer dans celui de l'écriture et de l'être. Dans l'usage courant que nous avons de la parole, nous portons peu attention aux mots que nous employons. Que nous racontions, commentions, répondions, informions, manifestions, la primauté que nous accordons à la clarté et à l'efficacité de notre discours restreint d'abord le nombre de mots auxquels nous recourons et les assujettit ensuite à l'idée que nous leur imposons de transmettre. Aussi bien ne les considérons-nous pas séparément, en eux-mêmes, ne prenons-nous pas le temps d'observer le faisceau de significations dont chacun serait susceptible de rayonner, ne nous soucions-nous pas même de les lester du poids du réel qu'ils ont charge d'évoquer : l'important est la signification globale que leur enchaînement dèlivie, laquelle n'est pas forcèment liée à eux de façon foncière, ne repose pas sur leur étymologie par exemple, ou leur morphologie; la preuve en est que, pour exprimer la même chose, nous aurions pu employer d'autres mots ou d'autres tournures, que nous avons pris ceux qui nous arrivaient sur la langue comme ils venaient, c'est-à-dire dictés par le contexte, par l'air du temps, voire par nos interlocuteurs et leur milieu. Au fond, nous n'avons pas parlé, c'est le langage qui a trouvé en nous une médiation pour jouer et circuler, ce sont les mots qui ont usé de nous pour retentir, tenant aussi peu compte de notre individualité que nous n'avons tenu compte de la spécificité de chacun d'eux.

Le propre de l'écriture est d'isoler les mots les uns des autres et d'en proposer une expérience qui n'est plus celle du discours, des concepts, des idées, du verbe, mais celle du signe, du symbole, de la parole. Encore qu'un texte soit pour une bonne part la transcription d'un discours qui pourrait être tenu oralement et obéisse par conséquent aux lois du langage, il dispose les mots de telle sorte qu'il y a toujours la possibilité de s'arrêter sur eux ou d'y revenir, de les examiner, de les interroger, d'être alerté par leur configuration or thographique ou phonétique qui a sa répercussion sur d'autres facultés en nous que celle de l'intelligence et nous donne de les appréhender, même si ce l'est de façon furtive et inexprimable, dans une autre dimension que celle de leur message abstrait.

Ceci ne s'inscrit certes pas dans la direction donnée à la littérature que l'on écrit et que l'on lit de plus en plus comme un enregistrement, à laquelle on demande de se démarquer le moins possible du parler contemporain, que l'on souhaite transparente, alerte, dẻliée, où l'on recommande d'effacer ce qui rappellerait précisément la progression tremblante et les stations méditatives de l'écriture. Il en resulte des ouvrages d'une uniformité de style, et partant d'inspiration, de contenu même, oủ l'habileté de l'auteur, voire le talent qui est vanté en lui, se mesure à la conformitè du tableau qu'il dresse de la société, à son rendu des comportements, sentiments et aspirations qu'il est convenu de tenir pour les nôtres, ou encore à la docilité de son imagination à se couler 
dans la mouvance, dans les stéréotypes et dans les procédés des modèles littéraires en vogue. Ie mot, en l'occurence, n'a pas valeur par rapport à sa propre histoire ni à sa capacité possible de surprendre, d'ouvrir sur l'inoui, d'apporter un sens comme venu d'ailleurs, d'alarmer sur la présence qui le traça et sur les raisons pour lesquelles elle le fit, de susciter une rencontre; il n'est qu'un référant renvoyant le lecteur à ce qu'il est entendu qu'il est et à l'idéologie ambiante.

Ecrire, pourtant, cest se mettre en situation d'être arrêté par les mots, soit en s'apercevant qu'aucun ne correspond exactement à la forme musicale et significative qui lui préexiste en nous, soit en « tombant », en cours de recherche, sur une « perle » qui nous éblouit, un vocable dont la physionomie stimule notre imagination jusqu'au vertige, un terme qui à lui seul est tout un livre. Ecrire, c'est se détacher du langage, ou le détacher de nous, pour le considérer comme un des èléments du monde au même titre que l'eau des rivières, les fleurs des champs, le sable des déserts et des plages. Un élément du monde qui dit le monde et nous-mêmes un peu à la façon dont le fracas des eaux dit les rochers qu'elles dévalent, dont le vent dit les branches qu'il secoue, dont les senteurs disent des choses l'invisible substance qui les déplie, dont la lumière dit les formes sur lesquelles elle s'appuie pour établir ses volumes. Des lors les mots, éléments de cet élément, variables selon les climats, les accidents, les Histoires, les corps, les gorges, ne sont pas appelés à former le plus lisse des miroirs ou à installer un artificiel jeu de reflets, ils sont les terminaisons extrêmes de racines qui remontent jusqu'à la nuit des Temps. Ecrire, C'est jouer sur ces extrémités sensibles comme des cordes ou comme les touches d'un clavier d'orgue. C'est apprendre, et savoir, tels les personnages des œuvres classiques, que chaque mot employé engage le destin de celui qui l'emploie de la même manière que le peintre sait que chaque coup de pinceau sur une toile engage avec son geste tout son être.

\section{III}

Même à l'oral, le langage prèsente une dimension sensuelle la plupart du temps oubliée ou négligee mais qui, lui étant inhérente, n'en persiste pas moins. Il suffit d'un lapsus, d'un dëfaut de prononciation, de la parabole hardie que dessinent certaines intonations, de la vigueur que le souffle particulier donne à une tournure, d'une syllabe déformée par le timbre d'une voix ou les fibres d'une sensibilité singulière pour appréhender le langage sous l'angle de la médiation révélatrice qu'il est aussi, a contrario en quelque sorte, par l'empreinte que sur lui laisse la personnalité irréductible du parleur. Autant que les émotions sur son visage, dans ses gestes, dans sa chair, le langage suscite la mélodie de sa peau, de ses cordes, de ses fibres; il prend dans sa voix un accent qui exprime la texture de ses muscles, la résonance de ses viscères, les arcanes de l'être animal et tangible. Sa voix est comme une vapeur qui 
exhale plus que son souffle et jusqu'aux sillons de son épiderme, jusqu'à la substance de ses cellules. Sans doute le sens des mots et le souci de leur en donner un sont-ils essentiels dans la mesure où sans eux il n'y aurait pas de ton, ni de timbre, ni de voix, mais, dans l'écriture, c'est ce ton, et ce timbre et cette voix qui, paradoxalement puisqu'on ne les y entend pas, deviennent essentiels, ce qui définit un écrivain tenant moins au contenu du texte qu'il écrit qu'à la manière dont il s'interroge, à l'attitude qu'il prend vis-à-vis du langage et pour embrasser le monde, au trajet enveloppant ou direct de son regard, à la disposition dont se contractent où se déploient ses traits, aux gestes du personnage qu'on devine sous le texte et qu'on ne trouverait peutêtre pas en le voyant et en l'écoutant. Iui-même se guide sur l'alerte tout intuitive que constituent les réactions de son corps : il sait qu'il « s'atteint » lorsqu'il peut imposer au langage un tempo qu'il sait correspondant au rythme distinctif de son métabolisme, de même qu'il doit aux leçons mystérieuses de son oreille interne le choix des phonemes qu'il retient, leur disposition dans la phrase, les harmoniques qu'il en tire. Le phrase d'un écrivain suppose que la seule place d'un adjectif dépend du rythme de sa respiration. Ce qui fait le prix de son œuvre est attaché aux précipitations et aux ondulations singulières de son rythme, chez l'un au caractère âpre de bouche pâteuse, chez un autre à la douceur de son murmure, chez un autre encore aux accents étincelants d'une aria, chez chacun à tout ce qui est traces inscrites de son corps et fait qu'un texte est geste, danse, chant. En somme, sur le papier sensible, l'instantané d'une présence sans laquelle l'écriture ne serait que de la typographie.

La critique scolaire et universitaire nous entraîne à une lecture biographique, psychologique ou idéologique plus qu'à une approche existentielle de la littérature. C'est à la perception de l'universel qu'elle prépare, non à une rencontre avec l'unique, le limité, le prochain, en quoi consistent pourtant la chance et le plaisir de la lecture. La critique vise a la compréhension de l'espèce humaine et de l'art, non à l'apprentissage de la lecture et au développement des facultés de finesse, de perspicacité et d'empathie qui permettraient, au fil d'un texte, de « balayer » les multiples registres sur lesquels l'écrivain joue simultanément. Sans doute suffit-il de lire un paragraphe d'un auteur pour le reconnaître, surtout quand il s'agit d'un Péguy, d'un Proust, d'un Claudel, d'un Cèline, ou même d'un San Antonio. Cela implique que la totalité d'un écrivain est d'emblée perceptible, mais un texte en appelle à une adhèsion toujours plus intime qui exige une perception à la fois plus étendue et plus aiguë: ce sont jusqu'aux déplacements du regard, aux mouvements du corps, aux élans et aux pauses du désir, aux vibrations de la sensibilité, à la nature unique de l'être, à la proximité de sa présence que, par la médiation de la fiction, un écrivain offre de remonter en lui. 


\section{IV}

Il est temps de passer a un exercice pratique. Voici cinq textes extraits d'cuvres à ce point reconnues qu'ils ont été proposés comme sujets de commentaire composé au baccalauréat. Ils ont pour point commun d'évoquer un paysage. Ils expriment la vision que chacun de leurs auteurs a de la nature et le rapport qu'ils entretiennent avec elle. L'expérience eut èté plus concluante si, comme dans les pastiches, ces cinq auteurs avaient pris le même coin de nature pour objet de description, mais cet objet, on va le voir, vaut moins pour lui-même que pour la matière qu'il offre à chacun pour, au même titre que les mots, déployer sa propre nature.

Les rives du lac de Bienne sont plus sauvages et romantiques que celles du lac de Genève parce que les rochers et les bois y bordent l'eau de plus près, mais elles ne sont pas moins riantes. S'il y a moins de cultures de champs et de vignes, moins de villes et de maison, il y a aussi plus de verdure naturelle, plus de prairies, d'asiles ombrages, de bocages des contrastes plus fréquents et des accidents plus rapprochès. Comme il n'y a pas sur ces heureux bords de grandes routes commodes pour les voitures, le pays est peu fréquenté par les voyageurs; mais il est intéressant pour les contemplatifs solitaires qui aiment à s'enivrer à loisir des charmes de la nature, et à se recueillir dans un silence que ne trouble aucun autre bruit que le cri des aigles, le ramage entrecoupé de quelques oiseaux, et le roulement des torrents qui tombent de la montagne. Ce beau bassin d'une forme presque ronde enferme dans son milieu deux petites illes, l'une habitée et cultivée, d'environ un demi lieu de tour, l'autre plus petite déserte et en friche et qui sera détruite à la fin par les transports de la terre qu'on en ôte sans cesse pour réparer les dégâts que les vagues et les orages font à la grande. Cest ainsl que la substance du faible est toujours employée au profit du puissant.

Jean-Jacques ROUSSEAU, Les Rêveries du promeneur solitaire, $5^{\varepsilon}$ promenade.

Au milieu de ces collines aux formes admirables et se précipitant vers le lac par des pentes si singulières, je puis garder toutes les illusions des descriptions du Tasse et de l'Arioste. Tout est noble et tendre, tout parle d'amour, rien ne rappelle les laideurs de la civilisation. Les villages situés à mi-côte sont cachés par de grands arbres, et au-dessus des sommets des arbres s'élève l'architecture charmante de leurs jolis clochers. Si quelque petit champ de cinquante pas de large vient interrompre de temps à autre les bouquets de châtaigniers et de cerisiers sauvages, l'œil satisfait y voit croittre des plantes plus vigoureuses et plus heureuses là qu'ailleurs. Par-delà ces collines, dont le faîte offre des ermitages qu'on voudrait tous habiter, l'œil étonné aperçoit les pics des Alpes, toujours couverts de neiqe, et leur austérité sévère lui rappelle des malheurs de la vie ce qu'il en faut pour accroître la volupté présente. L'imagination est touchée par le son lointain de la cloche de quelque petit village caché sous les arbres : ces sons portés par les eaux qui les adoucissent prennent une teinte de douce mélancolie et de résignation, et semblent dire à l'homme : La vie s'enfuit, ne te montre donc point si difficile envers le bonheur qui se présente, hâte-toi de jouir. Le langage de ces lieux ravissants et qui n'ont point de pareils au monde, rendit à la comtesse son cœur de seize ans.

STENDHAL, La Chartreuse de Parme. 
A Saint-Etienne, nous avons pris le train qui longe la Loire.

Jai toujours aimé les rivières!

De mes souvenirs de jadis, j'ai gardé par-dessus tout le souvenir de la Loire bleue ! Je regardais là-dedans se briser le soleil; l'écume qui bouillonnait autour des semblants d'écueil avait des blancheurs de dentelle qui frissonne au vent. Elle avait été mon luxe, cette rivière, et j'avais pêché des coquillages dans le sable fin de ses rives, avec l'émotion d'un chercheur d'or.

Elle roule mon cœur dans son flot clair.

Tout à coup, les bords se débrident comme une plaie.

Cest qu'il a fallu déchirer et casser à coups de pioche et à coups de mine les rochers qui barraient la route de la locomotive.

De chaque côté du fleuve, on dirait que l'on a livré des batailles. La terre glaise est rouge, les plantes qui n'ont pas été tuées sont tristes la végétation semble avoir été fusillée ou meurtrie par le canon.

Cette poésie sombre sait, elle aussi, me remuer et m'émouvoir. Je me rappelle que toutes mes promenades d'enfant par les champs et les bois aboutissaient à des spectacles de cette couleur violente. Pour être complète et profonde mon émotion avait besoin de retrouver ces cicatrices de la nature.

Ma vie a été labourée et mâchẻe par le malheur comme cet ourlet de terre griffèe et saignante.

Ah ! je sens que je suis bien un morceau de toi, un éclat de tes rochers, pays pauvre qui embaume les fleurs et la poudre, terre de vignes et de volcans!

Jules VALLĖS, Le Bachelier.

A la première haleine de la forêt, mon cceur se gonfle. Un ancien moi même se dresse, tressaile d'une triste allégresse, pointe les oreilles, avec des narines ouvertes pour boire le parfum.

Le vent se meurt sous les allées couvertes, où l'air se balance à peine, lourd, musque... Une vague molle de parfum guide les pas vers la fraise sauvage, ronde comme une perle, qui mûrit ici en secret, noircit, tremble et tombe, dissoute lentement en suave nourriture framboisée dont l'arôme se mêle à celui d'un chevrefeuille verdâtre, poissé de miel, à celui d'une ronde de champignons blancs... Ils sont nés de cette nuit, et soulèvent de leurs têtes le tapis craquant de feuilles et de brindilles... Ils sont d'un blanc fragile et mat de gant neuf, emperles, moites comme un nez d'agneau; ils embaument la truffe fraîche et la tubéreuse.

Sous la futaie centenaire, la verte obscurité solennelle ignore le soleil et les oiseaux. L'ombre impérieuse des chênes et des frênes a banni du sol l'herbe, la fleur, la mousse et jusqu'à l'insecte. Un écho nous suit, inquiétant, qui double le rythme de nos pas... On regrette le ramier, la mésange; on désire le bond roux d'un écureuil ou le lumineux petit derrière des lapins... Ici la forêt, ennemie de l'homme, l'écrase.

Tout près de ma joue, collé au tronc de l'orme où je m'adosse, dort un beau papillon crépusculaire dont je sais le nom : lykénēe... Clos, allongé en forme de feuille, il attend son heure. Ce soir, au soleil couche, demain, à 
l'aube trempée, il ouvira ses lourdes ailes bigarrées de fauve, de gris et de noir. Il s'épanouira comme une danseuse tournoyante montrant deux autres ailes plus courtes, éclatantes, d'un rouge de cerise mûre, barrées de velours noir; - dessous voyants, juponnage de fête et de nuit qu'un manteau neutre, durant le jour, dissimule...

COLETTE, Les Vrilles de la vigne.

Que d'heures passées à écraser les absinthes, à caresser les ruines, à tenter d'accorder ma respiration aux soupirs tumultueux du monde ! Enfoncé parmi les odeurs sauvages et les concerts d'insectes somnolents, j'ouvre les yeux et mon cœur à la grandeur insoutenable de ce ciel gorgé de chaleur. Ce n'est pas si facile de devenir ce qu'on est, de retrouver sa mesure profonde. Mais à regarder l'échine solide du Chenoua, mon cœur se calmait d'une ètrange certitude. J'apprenais à respirer, je m’intégrais et je m'accomplissais. Je gravissais l'un après l'autre des côteaux dont chacun me réservait une récompense, comme ce temple dont les colonnes mesurent la course du soleil et d'où l'on voit le village entier, ses murs blancs et roses et ses vérandas vertes. Comme aussi cette basilique sur la colline Est : elle a gardé ses murs et dans un grand rayon autour d'elle s'alignent des sarcophages exhumés pour la plupart à peine issus de la terre dont ils participent encore. Ils ont contenu des morts; pour le moment il y pousse des sauges et des ravenelles. La basilique Sainte-Salsa est chrétienne, mais chaque fois qu'on regarde par une ouverture c'est la mélodie du monde qui parvient jusqu'à nous : côteaux plantés de pins et de cyprès, ou bien la mer qui roule ses chiens blancs à une vingtaine de mètres. La colline qui supporte SainteSalsa est plate à son sommet et le vent souffle plus largement à travers les portiques. Sous le soleil du matin, un grand bonheur se balance dans l'espace.

Albert CAMUS, Noces.

Les affinités et les différences sensibles entre ces cinq auteurs s'expliquent peu par la diversité des paysages qu'ils décrivent, encore qu'ils soient significatifs; elles ne sont pas à chercher non plus, de facon spécifique, dans les contextes historiques où ils s'inscrivent, encore que ces contextes ne manquent pas de dèterminer une part du vocabulaire, de la syntaxe et, partant, de la vision. A peu de choses près on retrouve les mêmes éléments dans la matière dont ils usent : l'eau, la verdure, les sons, la lumière, la solitude; et si, d'un texte à l'autre, les mots ne sont pas les mêmes, ils appartiennent aux mêmes registres, jusqu'à ce recours, en chacun, à des noms propres.

C'est à des particularités plus constitutives que se rattachent les différences, aux attitudes symptomatiques vis-à-vis du langage, aux énergies singulières dont ces attitudes elles-mêmes tēmoignent, aux natures des sensibilités qui président au choix des mots mais surtout à la manière d'en susciter la morphologie évocatrice par leur emplacement dans la phrase, par leurs rapprochements ou leurs écarts. Point n'est besoin d'entrer dans des considérations stylistiques très précises pour repérer que l'ordonnancement des mots 
est déterminé par l'ordonnancement de la description elle-même, qui suit le trajet du regard, lequel n'est pas tant celui des yeux (aucun de ces paysages n'est peint d'apres nature, tous sont reconstruits par l'écriture) que celui d'une sensibilitè distinctive.

Rousseau, Camus, Colette abordent le motif par la périphèrie et s'acheminent en son centre par les contours en un mouvement englobant, en un soigneux travail d'approche, soucieux d'atteindre au secret de la séduction que produisent sur eux les apparences et de s'y identifier ou de s'y fondre : la petite île au centre du lac de Bienne, le lykénée au plus sombre de la forêt, le sommet de la colline où vibrent les soupirs tumultueux du monde. Stendhal explore un canton idéal, né de souvenirs litteraires et construit au fond de lui comme l'image d'un paradis : son écriture rapide procède par à-plats désinvoltes qui suffisent à évoquer un cadre que des touches ensuite, distribuées de bas en haut et du centre vers les lointains, complètent. Quant à Jules Vallès, son évocation procède, à l'instar de l'eau de la Loire qui se fracasse contre les pierres et du choix de mots courts, de préférence monosyllabiques, par diffraction, par jaillissements erruptifs, par éclats qui s'irisent, qu'ils concernent l'objet décrit, des souvenirs fulgurants, ou des déchirures de l'être.

D'autres points de vue révéleront d'autres composantes et regrouperont les auteurs de façon différente: Chez Rousseau et Stendhal, le paysage incarne un rêve de retraite, de recès, d'abri; il s'approprie les qualités traditionnellement attribuées à la mère, il en reproduit les formes et le giron, il est liè à l'enfance; la sagesse énigmatique que lui suppose sa sérénité inspire à l'écrivain, incidemment, quelques notations d'ordre moral. La description de Camus en comporte aussi, qui semblent lui être dictées par l'èdifiante disparité entre la toute puissance du réel impassible et la vanité des ambitions humaines, sur quoi insiste l'ensemble de son évocation; la nature ne lui est pas refuge mais divinité suprême, détentrice de la seule vérité qui compte, et l'homme vient la consulter comme un oracle. Vallès et Colette pour leur part s'y enfoncent, s'y ressourcent, y retrouvent le visage perdu de leur être comme en un miroir, l'un en se rappelant, à la vue de la terre bouleversée de sa patrie, sa propre nature, sauvage, farouche, domptée à force de volonté et d'assujettissements, l'autre en s'y perdant comme dans les couches les plus enfouies de la conscience, au milieu de ses désirs les plus immémoriaux, avant d'y atteindre la certitude de ce qu'elle est et de ce à quoi elle est promise.

La prose de cette dernière est la plus sensuelle, elle tend à se rapprocher des ondulations voluptueuses et voraces d'un fëlin, elle feutre les sonorités, amatit les éclats, fait sourdre des mots ces senteurs et ces saveurs qu'ils sont le moins propres à traduire. Rousseau n'en est pas si éloigné quoique la sensualité chez lui soit moins pénétrante et se borne à l'ouverture des sens; son écriture n'en cherche pas moins l'enveloppement et les stimuli berceurs de 
la caresse. Le style de Camus est sous-tendu par le ressort d'un questionnement d'ordre spirituel qui le retient d'adhérer tout à fait à son objet mais on le sent près de délaisser les concepts au profit de termes plus concrets, plus proches de la matière et du frémissement des choses, sous la pulsion d'un désir qui porte l'auteur à une rencontre physique, à la nudité, à l'êtreinte. L'écriture éclatée de Vallès approche la brutalité et la violence de la matière en train de prendre forme à coups de pioche et d'explosions; c'est un chaos qui s'ordonne, une gerbe dont les jets se figent en une irradiation qui a la cohérence à la fois arbitraire et rigoureuse des cristaux. l'évocation de Stendhal a la légèreté de sa plume empressée; ses mots puisès dans le langage courant dressent un paysage d'estampe dont la force évocatrice tient a l'économie des traits utilisés; il est parmi les cinq auteurs l'écrivain le moins soucieux d'écriture, le plus rebelle à la rature, il ne peut écrire que sous l'urgence, il ne s'y passionne que dans le feu de l'action.

Ces textes recréent des paysages que nous n'aurons jamais la possibilité de voir autrement qu'en les lisant. Ils arpentent des territoires qui sont ceux de la solitude. Ils racontent des singularités qui aspirent à plus de singularité encore. Ils explorent ce qui ne pourrajamais être pour nous que des possibles. Ils ne parlent pas du monde, ni de nous. Ils posent sur ce qui leur reste de l'autre un regard qui demeure le leur, et c'est dans ce face à face tendant sans fin à se résoudre en étreinte que le lecteur a quelque chance de sentir une parenté avec la situation oủ lui-même se trouve, solitaire face au monde solitaire.

\section{V}

L'écriture condamne à n'employer aucun mot qui ne soit « parole », c'està-dire qui non seulement corresponde à un signifié mais qui porte en soi une énergie, un èlan, une histoire, un regard, une sensibilité. Dire n'est pas uniquement l'objet de l'écriture créatrice et ne suffit pas, en tout cas, à réaliser une œuvre. Aussi bien l'écriture n'est-elle pas à proprement parler une vocation, un destin. On ne naît pas écrivain. Qui le devient possède au départ des donnèes semblables à celles de tout un chacun et pas propres davantage à l'orienter vers l'écriture. Si celle-ci se propose à lui comme la médiation la mieux à même de répondre à son desir de creer, elle ne correspond pas forcément le mieux aux moyens dont il dispose, mais il ne devient écrivain, en tout état de cause, qu'au fur et à mesure qu'en la pratiquant il découvre l'écriture, la considère pour ellemême, en use comme d'une matière.

Ce qui rend un texte irremplaçable ne relève pas de son signifié, ni même des intentions de son auteur. Ce signifiè et ces intentions constituent également le fonds commun de toute vie humaine. Quoi de plus partagé que les émotions, les impressions, les sentiments, les appétits, les passions, les 
angoisses, les douleurs, les cruautés? Quant aux idées que les écrivains s'épuisent à promouvoir ou à défendre jusqu'à en mourir, elles sont destinées à vieillir, à entrer en désuétude, à se révéler surannees. Triste sort que celui de ces pensées dont nous ne pouvons concevoir qu'elles apparaissent un jour aussi artificielles, incommodes, puériles, ridicules, que nous le semblent les costumes et les poses des siècles passés. Elles nous semblent à ce point constitutives de nous qu'il nous est impossible de supposer qu'elles soient des conventions ou des chimères promises au rebut en même temps que nos coiffures, nos tenues vestimentaires, nos gestes, nos mœurs. Et cependant ce n'est pas plus sur elles que sur ses perruques poudrées que la prose de Rousseau doit de perdurer, non plus que celle de Montaigne ne le doit à ses fraises et à ses pourpoints, celle de Racine à ses talons et à ses jabots, celle de Vallès au romantisme ou celle de Camus à la littérature engagée. Ces écrivains ne doivent de l'être qu'à la forme, inouie avant eux et qui restera inimitable, donnée à la matière du langage par les tensions particulières et indicibles de leur être, la rendant capable d'exprimer quelque chose d'unique, c'est-à-dire d'éternellement nouveau. Un écrivain ne l'est qu'à proportion de l'évidence qu'il sait donner à sa solitude qui elle, souvent sinon toujours a l'encontre de ses convictions, s'oppose aux conventions par lesquelles une société endigue le désir individuel qui la menace, voire dénonce le scandale du vécu, les contradictions de la nature humaine, le conflit entre ses aspirations divergeantes, les limites qui la contraignent.

Comme toute matière quand on la travaille, l'écriture présente des faiblesses et des résistances qui en viennent à occuper l'écrivain bien plus que le sujet ou le motif dont il était parti. Les mots y sont sollicités dans leur signification que l'on force, que l'on modèle, dont on joue en vue d'obtenir un effet; ils le sont aussi dans leur coloration auditive, leur charge de sonorités, leur rythme. Quand j'écris : achillée, aspidistra, basalmine, bergamote, camomille, cinnamome, coronille, rossolis, germandrée, jacée, jonquille, jusquiame, salsepareille, origan, valériane, zinnia, je désigne par chacun de ces mots une fleur ou une plante précise qui, dans le réel, a sa couleur, sa forme, son odeur, ses propriétés. Dans un texte, cette référence au rẻel n'a qu'une valeur secondaire, et d'ailleurs personne n'ira y voir; l'essentiel est le paysage sonore, le bouquet de vie immobile et purement littéraire, l'atmosphère d'indèfinies correspondances obtenue par le recours à ces phonèmes qui, en outre, auront été sélectionnés non en raison de la présence indubitable de ce qu'ils désignent dans une portion de nature réelle mais pour les harmoniques engendrées par leur assortiment : il pouvait y avoir de la moutarde, aussi, et des pissenlits, des gueules-de-loup : si leurs frottis colorés sont beaux dans la réalité, il n'est pas dit que leurs termes s'accordent avec les autres termes retenus.

Les multiples nuances que permettent l'abondance du vocabulaire, les complexités de la syntaxe, les surprises de sa logique ou de ses aberrations 
conduisent à la découverte d'un autre sens que celui qu'on lui aurait spontanément confié de transmettre. Elle surprend, elle piège, elle contraint à poser des règles qui elles-mêmes obligent à des développements imprévisibles, imprévus. On y avance sur un chemin aux embranchements proliférants, on y fait fausse route, il faut accepter de revenir sur ses pas, on aborde des zones toujours plus dangereuses.

L'écriture a ses limites aussi : sa lenteur, ses rigiditès, l'inadéquation inéluctable de ses mots. On ne traite avec elle qu'en rusant, on la force par des équivalences, des périphrases, des transpositions qui répercutent très loin leurs conséquences, jusqu'à tout bouleverser. La difficultè que l'on peut avoir, par exemple, à décrire le geste d'un personnage, dans un roman, amène à lui donner un autre geste, mais c'est tout le personnage qui en est modifié, et la nouveauté qu'alors il révèle a ses répercussions dans ses rapports avec les autres personnages qui eux-mêmes en sont changés, et ainsi de suite. Il en va de même, et de façon plus cruciale encore, de l'écrivain sur qui l'écriture exerce de telles contraintes qu'il lui arrive d'écrire le contraire de ce qu'il pense ou croit penser. Il se doit de constater, avec émerveillement ou terreur, que le démiurge qu'il devient grâce à l'écriture n'est qu'une dimension ignorée ou un possible de soi.

\section{VI}

S'il apparaît dans les mots que l'écrivain n'est qu'à la poursuite de luimême, ce sont les mots qui le font apparaître mais ce qui importe reste ce vers quoi ils avaient été lancés. Un écrivain décrit le spectacle des vagues le long de la plage : sous la plume d'un autre le même spectacle serait exprimé en des termes différents qui ne se révéleraient pas moins efficaces. Ce qu'un écrivain écrit, comme ce qui l'obsède, c'est par conséquent lui-même, et lui seul. Mais tel n'était pas l'objet de l'intention qui l'a porté vers l'écriture : la mer seule l'intéressait. Quand même ne retrouvons-nous que lui dans son écriture, le réel s'y trouve aussi, en dédicace.

L'écriture veut moins arrêter sur ce qu'elle dit qu'orienter vers ce qu'elle n'a pas pu dire, pareillement au tableau dans lequel ce ne sont pas les lignes et les volumes dessinés qui importent en eux-mêmes mais la vibration qui s'en dégage, l'espace qu'ils soulignent et, en dernier ressort, le regard qui les regarde. Tel est le paradoxe de l'écriture crèatrice que, si elle renvoie l'écrivain à luimême, il ne saurait s'en passer pour approcher l'au-delà de soi, et que plus grande est sa maîtrise d'expression, plus proche est-il de ce qui habite le silence. Les anecdotes qu'il raconte, d'ailleurs inventées ou travesties, ne suffisent à remonter son ressort qui ne peut l'être que par ce que la fiction lui offre de plus vrai pour le vivre. L'écriture ne tend qu'à épuiser le dictionnaire. 
Ce qui distingue le créateur, n'est-ce pas l'audace ? Une sorte de culot. Il y a, par exemple, dans les arabesques des violons de telle partie du Messie de Hændel, des coups de crayon d'une désinvolture qui défie la raison : on tremble pour Hændel. A tort, bien sûr : l'œuvre est achevée, la mèlodie est fixée, elle est ce qu'elle devait être. Mais elle m'a ébranlé, et je suis frémissant encore du risque énorme que le compositeur avait pris quand il lança son geste, risque auquel je suppose les musiciens à chaque fois exposés de nouveau quand ils l'interprētent. Même audace dans le domaine de la parole. De dire : " Je vois la mer briller » a dire : « la mer brille », ne s'opère pas un effacement de ma subjectivité : je m'expose à tous les démentis. Mais combien davantage encore m'expose-je à la faillite en affirmant, ce qui est le dépassement de la poésie : «la mer regarde. »

Comme en ces moments d'illumination ou c'est le monde qu'avec une acuité qui nous dépouille de toute défense nous rencontrons dans la vibration d'un mot, la parole créatrice engage à prendre tous les risques. Que va trouver l'écrivain au bout de ses phrases ? Il ne peut le savoir à l'avance : ce n'est pas qu'il vit puis écrit ce qu'il a vécu. Il vit et ensuite, par l'écriture, crée ce qu'il vit. Il le « réalise » en l'inscrivant : en même temps il se rend compte et il accomplit. Dans la parole, cest un moi en avance sur moi qui s'exprime, un moi que je suis mais ne sais point être encore. En ce sens, si l'œuvre d'un écrivain le dépasse, c'est-à-dire si elle l'engage au-delà de ce qu'il peut, dans les limites qui sont les siennes, inactuellement et universellement contenir, il ne saurait être par elle réalisé. En elle-même, l'œuvre ne constitue pas un accomplissement. La vocation foncière de l'écrivain n'est pas de dire mais d'écouter. L'écriture ne tend qu'à èpuiser le dictionnaire.

\section{Ecrivain}

Paris 